\title{
Study of Structural Changes of Water-Based Magnetic-Fluid by Acoustic Spectroscopy
}

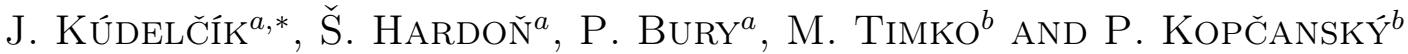 \\ ${ }^{a}$ Department of Physics, University of Žilina, Univerzitná 1, 01001 Žilina, Slovakia \\ ${ }^{b}$ Department of Magnetism, IEP SAS, Watsonova 47, 04001 Košice, Slovakia
}

\begin{abstract}
The effect of an external magnetic field on the changes in structural arrangement of magnetic nanoparticles in water based magnetic fluid was studied by acoustic spectroscopy. When a magnetic field is increased, the interaction between the magnetic field and the magnetic moments of nanoparticles leads to the orientation of magnetic nanoparticles and their following aggregation to long chains that cause the increase of acoustic attenuation. The attenuation of acoustic waves measured for jump changes of the magnetic field to 100, 200, and $300 \mathrm{mT}$ at temperature $20^{\circ} \mathrm{C}$ showed that the changes of acoustic attenuation increased slowly to a stabilized state that after switching off the magnetic field decreased immediately to initial value. The dependence of attenuation of acoustic waves at constant magnetic field on angle between the wave vector and direction of the applied magnetic field (attenuation anisotropy) has been measured, too. The measured anisotropy of acoustic attenuation attested structural changes of magnetic fluid in the magnetic field.
\end{abstract}

DOI: 10.12693/APhysPolA.131.919

PACS/topics: 75.50.Mm, 75.75.Jn

\section{Introduction}

Water based magnetic fluid has a wide application in areas of biomedicine, from diagnostics to treatment of diseases. In ideal model of magnetic fluid the magnetic nanoparticles interact with magnetic field and interaction with neighbors can be described by the coupling constant, which is function of temperature, the hydrodynamic diameter and magnetic moment of magnetic nanoparticles. The result of these interactions is new structures, which are dependent mainly on the value of applied magnetic field and type of magnetic fluid. The acoustic spectroscopy is very useful for the study of these structural changes in the external magnetic field and better information about types of structures can be obtained from the study of the anisotropy of acoustic attenuation [1-7].

\section{Models for structural arrangement}

In nowadays there are three main theoretical models describing structures arrangement of magnetic nanoparticles in a magnetic field [1-3]. Under the effect of an external magnetic field the nanoparticles of magnetic fluid become arranged into chains along magnetic field direction or clusters with various shapes. The study of structure properties of magnetic fluids in the magnetic field by the anisotropy of acoustic spectroscopy is useful tool for the better understanding about numbers and size of magnetic nanoparticles in structures.

The first theoretical description of the anisotropy of acoustic attenuation of magnetic fluid (MF) under an

*corresponding author; e-mail: kudelcik@fyzika.uniza.sk external magnetic field assuming that magnetic nanoparticles form the spherical clusters of some radius was published in 1986 by Taketomi [1]. These clusters form long chains, aligned in magnetic field direction with two types of motion: the rotation motion and the translation motion. These motions are activated by the acoustic field with dissipating the energy of the acoustic wave into heat. Taketomi derived the formula for calculations of the acoustic attenuation on the basis of these motions and using coefficients regarding of nanoparticles structures in magnetic liquids [1, 4].

The second similar theory is from Shliomis et al. [2], in their model the magnetic fluid contains mainly single particles (monomers) and smaller oligomers: dimers, trimers, etc. Their distribution shifts towards to higher oligomers in applied magnetic field that means that the number of nanoparticles in a chain slightly increases and any clusters are created. In this theory there is predicted only translation motion of chains together with some effect of vibration. This theory also follows that when the magnetic field is aligned with the acoustic waves, the absorption is maximal and monotonic decreases with angle to minimal values for perpendicular orientation. The decrease of acoustic attenuation is dependent on the degree of the anisotropy $S$, which is function of coupling constant and the applied magnetic field, and of the angle of anisotropy $[2,4]$.

Another interpretation of acoustic attenuation in MFs is based on the theoretical model proposed by Ahuja and Hendee [3]. In this model it is assumed that magnetic nanoparticles aggregates in the external magnetic field take form of prolate ellipsoids with the same size at given magnetic field. Major axis with length $b$ is oriented along the field lines and minor axis has length $a$. For higher distance of ellipsoids acoustic attenuation anisotropy arises only from the shape of aggregates. The attenuation coef- 
ficient of ultrasound wave in magnetic field derived in [3] and also used in [5] depends on the shape factor $K$ and the inertia coefficient $L$. These parameters mainly depend on the particles size and orientation to the acoustic field.

\section{Experimental}

The acoustic attenuation in the water based biocompatible magnetic fluid with doubled layered surfactant shell was studied. The mean diameter of a magnetic nanoparticle $\left(\mathrm{Fe}_{3} \mathrm{O}_{4}\right)$ and its standard deviations equal to $d_{m}=6.23 \pm 0.07 \mathrm{~nm}$ and $\sigma_{m}=2.72 \pm 0.03 \mathrm{~nm}$. The hydrodynamic size distribution of magnetic nanoparticles coated with oleate sodium in suspension was determined by differential centrifugal sedimentation and the mean hydrodynamic diameter and its standard deviation is $d=27.7 \pm 0.3 \mathrm{~nm}$ and $\sigma=24.1 \pm 0.3 \mathrm{~nm}[6]$. The coupling constant for the sample studied is $\lambda=1.18$. The shear viscosity is $6.4 \mathrm{mPa}$ s and the density of magnetic fluid was $1043 \mathrm{~kg} / \mathrm{m}^{3}$.

The magnetic fluid was placed in the thermostated closed measuring cell with temperature $20 \pm 0.1^{\circ} \mathrm{C}$ and the distance between two piezoelectric transducers was $0.9 \mathrm{~cm}$. The changes in the ultrasonic wave attenuation were measured by the pulse method based on measurement of intensity of the ultrasonic pulse passed through or reflected by the medium studied [2]. The frequency of the ultrasonic wave was $49 \mathrm{MHz}$.

\section{Results and discussion}

The dependence of acoustic attenuation on magnetic field for water-based magnetic fluid with olean sodium at $20^{\circ} \mathrm{C}$ shows a hysteresis effect (Fig. 1) [4, 7]. Similar effect was also observed also at other temperatures. The change of acoustic attenuation with increase of magnetic field is minimal until to $70 \mathrm{mT}$. The next increase of acoustic attenuation with magnetic field originated from the process of particle agglomeration and chains formation. The process of structural changes can even continue with the decrease of magnetic field and the acoustic attenuation still increase until to $160 \mathrm{mT}$. From this value of magnetic field the attenuation slowly decreases to initial value.

Figure 2 illustrates the acoustic attenuation change for three different step changes of the magnetic field to 100 , 200 , and $300 \mathrm{mT}$. At the beginning of the measurement the magnetic field was set to zero value and after that was applied its step change. The magnetic field was held at the constant value for $30 \mathrm{~min}$. Although the development of acoustic attenuation depends on the value of magnetic field, the initial quick change after the magnetic field is evident for all cases. At $100 \mathrm{mT}$ step change of magnetic field the attenuation slowly increases during whole time without reaching the stable value. For $200 \mathrm{mT}$ and $300 \mathrm{mT}$ step change the attenuation again increases to maximum and reaches the stable value after $15 \mathrm{~min}$. From the measurements of acoustic attenuation it could be seen that processes of agglomeration of

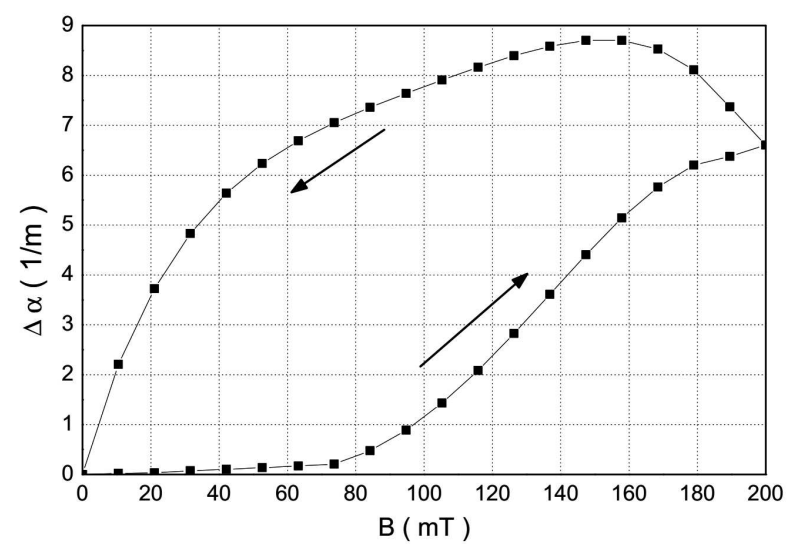

Fig. 1. The dependence of acoustic attenuation changes in linear change of the external magnetic field to $200 \mathrm{mT}$ with time step $3.3 \mathrm{mT} / \mathrm{min}$.

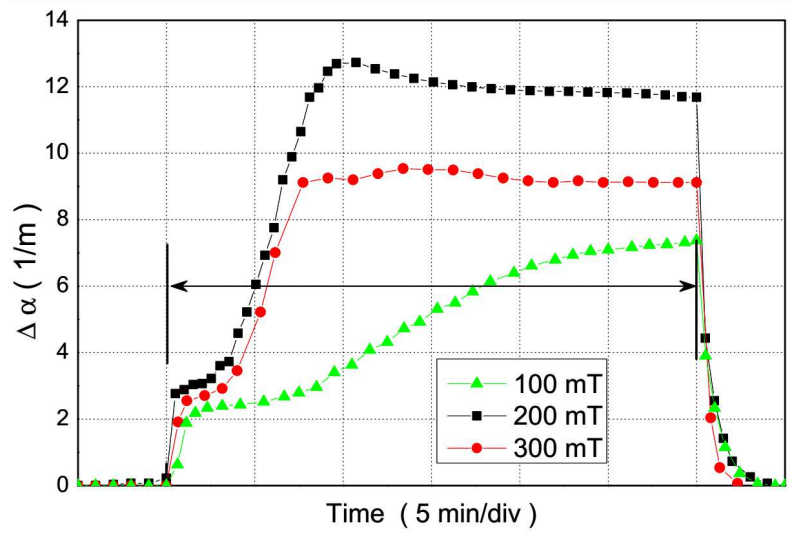

Fig. 2. The dependence of acoustic attenuation for jump change of the magnetic field to values: 100, 200, and $300 \mathrm{mT}$.

nanoparticles into oligomers or chains are dependent on the value of magnetic field and time $[7,8]$. For smaller magnetic field $(100 \mathrm{mT})$ the process of structural changes is twice longer than at higher fields. Smaller change of attenuation at $300 \mathrm{mT}$ comparing to $200 \mathrm{mT}$ is probably caused by bigger structures, which had higher sedimentation and finally cause the lower concentration. After the magnetic field was switched off, the acoustic attenuation decreased down exponentially with the relaxation constant about $80 \mathrm{~s}$. That means that the chains were very quickly disintegrated by thermal Brown motion and interactions between nanoparticles in chains are weak.

The anisotropy of acoustic attenuation (the dependence on the angle $\varphi$ between wave vector $\boldsymbol{k}$ and the magnetic field $\boldsymbol{B}$ ) measured at the constant value $200 \mathrm{mT}$ is depicted in Figs. 3 and 4. There is evident monotonic decrease of attenuation with the anisotropy angle. In the case of transformer oil-based magnetic fluids [4, 8] where nanoparticles created big clusters, the anisotropy had development with a maximum at the angle around $45^{\circ}$.

Applying the theoretical predictions of Taketomi [1] to our anisotropy results, the rotation and translation 
components, $\alpha_{\text {rot }}, \alpha_{t r}$ of acoustic attenuation were calculated. They are shown, together with measured data of the anisotropy in Fig. 3. The translation component of the acoustic attenuation $\alpha_{t r}(\varphi)$ is dominant and decreases monotonously with the increase of angle. In the case of the second component of the acoustic attenuation, $\alpha_{\text {rot }}(\varphi)$ is almost independent of the angle $\varphi$, which means symmetrical structures - cluster (more than 10 nanoparticles arranged into sphere). Using this theory we determined the clusters radius $107 \mathrm{~nm}$, constant of recovering force 1.13 and the volume concentration of structures $0.015 \%$.

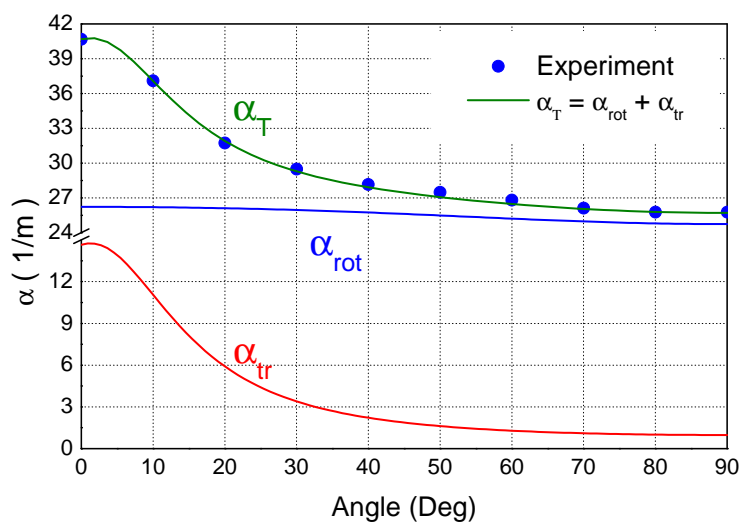

Fig. 3. Anisotropy of the acoustic attenuation measured at $B=200 \mathrm{mT}$ and its analysis using the Taketomi function with the development of individual components of $\alpha_{\text {rot }}$ and $\alpha_{t r}$.

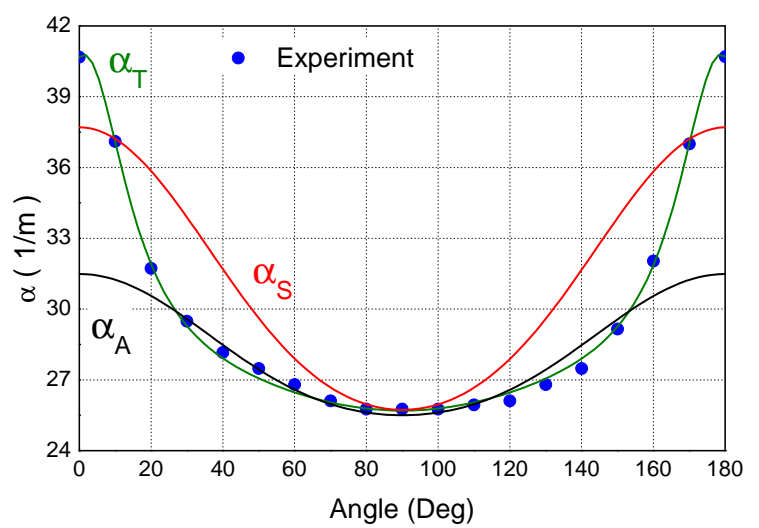

Fig. 4. Comparison of theoretical results of various theories with experimental results $\left(\alpha_{S}\right.$ - the Shliomis and Mond model, $(S=0.11), \alpha_{A}$ - the Ahuja and Hendee theory $(b=4 d, a=d)$ and $\alpha_{T}$ - Taketomi theory).

Figure 4 compares theoretical assumptions from the Shliomis et al. $\left(\alpha_{S}\right)$, Ahuja and Hendee $\left(\alpha_{A}\right)$ and Taketomi model $\left(\alpha_{T}\right)$ with experimental acoustic attenuation anisotropy measured at temperature $20^{\circ} \mathrm{C}$. As it can be seen from Figs. 3 and 4 the resultant theoretical curves are similar, all of them have monotonic decrease with the angle. The presented curve $\alpha_{A}$ is calculated by the theory of Ahuja and Hendee for prolate ellipsoid with major axis $b=4 d$, minor axis $a=d$ and density of aggregates $2460 \mathrm{~kg} / \mathrm{m}^{3}$ [5]. This theoretical model, that supposes only thin chains, does not well agree with experimental results. For the case when the minor axis equals twice of the hydrodynamic size of nanoparticle $(a=2 d)$, theoretical fit is much better and it is almost identical with the Shliomis prediction, which supposed only simple oligomers. However Ahuja and Hendee model does not take into account all mechanisms leading to the attenuation of acoustic wave in magnetic field, comparing with other models we can say that it is responsible for description of anisotropy of acoustic attenuation for these types of magnetic fluids. From the analysis obtained results using theoretical calculations of three different models we could say that magnetic nanoparticles in the investigated water based magnetic liquid create thick short chains prolate ellipsoids.

\section{Conclusions}

The attenuation of the acoustic waves propagating in the magnetic fluid consisting of magnetite nanoparticles suspended in water for various times development of the applied magnetic field were studied. The results have shown that this type of magnetic fluid need higher magnetic field to create structures. From the Taketomi model indicated that there was the important role of translation motions of clusters. Using appropriate next models the shapes of structures - prolate ellipsoid aligned with the magnetic field were determined.

\section{Acknowledgments}

This work was supported by VEGA project 2/0045/13 and the R\&D operational program Centrum of excellence of power electronics systems and materials for their components, No. OPVaV-2008/2.1/01-SORO, ITMS 26220120003 funded by European Community.

\section{References}

[1] S. Taketomi, J. Phys. Soc. Jpn. 55, 838 (1986).

[2] M. Shliomis, M. Mond, K. Morozov, Phys. Rev. Lett. 101, 074505 (2008).

[3] A.S. Ahuja, W.R. Hendee, J. Acoust. Soc. Am. 63 , 1074 (1978).

[4] J. Kúdelčík, P. Bury, P. Kopčansky, M. Timko, J. Magn. Magn. Mater. 388, 28 (2015).

[5] T. Hornowski, A. JĂłzefczak, B. Kołodziejczyk, M. Timko, A. Skumiel, M. Rajnak, J. Phys. D Appl. Phys. 48, 175303 (2015).

[6] A. Józefczak, T. Hornowski, V. Závišová, A. Skumiel, M. Kubovčiková, M. Timko, J. Nanopart. Res. 16, 2271 (2014).

[7] A. Józefczak, A. Skumiel, M Łabowski, J. Mag. Mag. Mat. 252, 365 (2002).

[8] J. Kúdelčík, P. Bury, J. Drga, P. Kopčanský, V. Závišová, M. Timko, Acta Phys. Pol. A $\mathbf{1 2 1}$ 1169 (2012) 